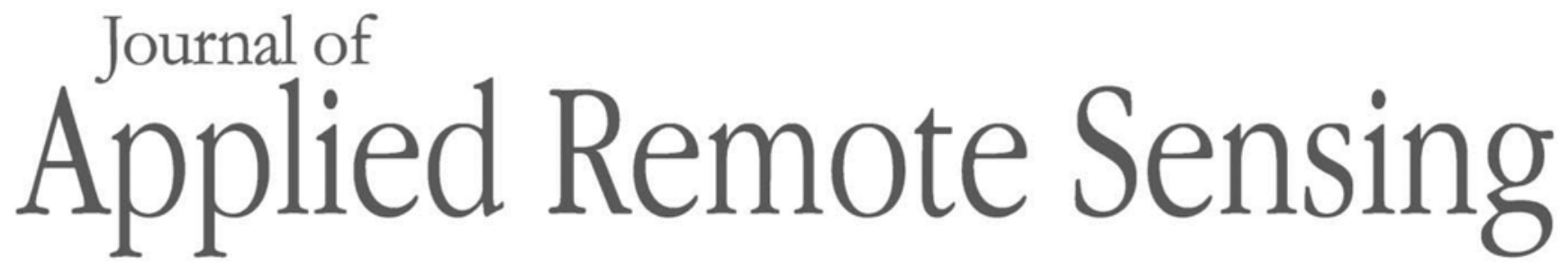

RemoteSensing.SPIEDigitalLibrary.org

\title{
Inverse synthetic aperture radar imaging of targets with complex motion based on the local polynomial ambiguity function
}

\author{
Qian Lv \\ Tao $\mathrm{Su}$ \\ Jibin Zheng
}




\title{
Inverse synthetic aperture radar imaging of targets with complex motion based on the local polynomial ambiguity function
}

\author{
Qian Lv,* Tao Su, and Jibin Zheng \\ Xidian University, National Laboratory of Radar Signal Processing, \\ 2 South Taibai Road, Xi'an 710071, China
}

\begin{abstract}
In inverse synthetic aperture radar (ISAR) imaging of targets with complex motion, the azimuth echoes have to be modeled as multicomponent cubic phase signals (CPSs) after motion compensation. For the CPS model, the chirp rate and the quadratic chirp rate deteriorate the ISAR image quality due to the Doppler frequency shift; thus, an effective parameter estimation algorithm is required. This paper focuses on a parameter estimation algorithm for multicomponent CPSs based on the local polynomial ambiguity function (LPAF), which is simple and can be easily implemented via the complex multiplication and fast Fourier transform. Compared with the existing parameter estimation algorithm for CPS, the proposed algorithm can achieve a better compromise between performance and computational complexity. Then, the high-quality ISAR image can be obtained by the proposed LPAF-based ISAR imaging algorithm. The results of the simulated data demonstrate the effectiveness of the proposed algorithm. () The Authors. Published by SPIE under a Creative Commons Attribution 3.0 Unported License. Distribution or reproduction of this work in whole or in part requires full attribution of the original publication, including its DOI. [DOI: 10.1117/1.JRS.10.015019]
\end{abstract}

Keywords: inverse synthetic aperture radar; cubic phase signal; local polynomial ambiguity function.

Paper 15698 received Oct. 4, 2015; accepted for publication Feb. 29, 2016; published online Mar. 18, 2016.

\section{Introduction}

The inverse synthetic aperture radar (ISAR) imaging technique for moving targets has attracted the attention of many radar researchers in the past three decades due to its significance in both civil and military applications. ${ }^{1-6}$ Generally, in order to obtain a well-focused ISAR image, the first procedure is to implement motion compensation, which includes range alignment (the translational and rotational migrations) and phase adjustment (the Doppler phase caused by the translation). ${ }^{6}$ Then the conventional range-Doppler (RD) algorithm can be used to generate a focused ISAR image, which indicates that, during the coherent integration interval, the Doppler frequency shift must remain constant. However, instances of targets that are usually uncooperative and engaged in complicated maneuvers often occurs. ${ }^{7-14}$ In this case, the RD algorithm does not work well for the time-varying characteristic of Doppler frequency. To deal with this problem, many articles have developed different solution for different degree of maneuverability, and they can fall into two categories: linear frequency modulation (LFM) signal model ${ }^{6-9}$ for targets with smooth motion and cubic phase signal (CPS) model $^{2,4,5,10-14}$ for targets with complex motion. For the slow-maneuvering target, the assumption that the azimuth echo is characterized as LFM signal is appropriate and practical, and has been demonstrated by Ref. 6 with experimental ship data. Many representative algorithms based on LFM signal model have been proposed, such as Radon Wigner transform, ${ }^{3}$ the modified Wigner-Ville distribution (WVD), ${ }^{6}$ the stretch keystoneWigner transform, ${ }^{7}$ the Lv's distribution, ${ }^{8}$ and the fractional Fourier transform. ${ }^{9}$ For targets with complex motion, in which rotational motion may cause higher-order phase terms, the traditional LFM signal model is not appropriate. For a more reasonable and accurate approximation, many

*Address all correspondence to: Qian Lv, E-mail: lvqian@stu.xidian.edu.cn 
articles have characterized the azimuth echo in a range bin as multicomponent CPSs, which is identified to be much more appropriate than the LFM model. ${ }^{10-14}$

With regard to the parameter estimation of CPS, numerous algorithms, including the cubic phase function (CPF) ${ }^{15,16}$ the higher-order ambiguity function, ${ }^{17}$ the product generalized CPF (PGCPF),${ }^{18}$ the product high-order matched-phase transform,,${ }^{19}$ and the modified version of CPF (MCPF) ${ }^{14}$ have been addressed, but all of them involve multilinear transformation and discrete Fourier transform for the nonuniformly spaced signal sample, which result in extensive crossterms under multi-CPSs and undesirable computational complexity. ${ }^{10-14,18,19-23}$ In Ref. 20, the parameter estimation algorithm based on the local polynomial Wigner distribution (LPWD) is proposed and has been successfully applied to ISAR imaging. ${ }^{21,22}$ However, after compensating the third-order term with the estimated parameter, the LPWD algorithm estimates the secondorder coefficient using CPF method, which does lead to the heavy computational burden due to the Fourier transform with respect to the nonuniformly spaced data. In general, the performance and computational cost are conflicting requirements, e.g., the modified discrete chirp Fourier transform for CPS can obtain robust performance against noise and handle multi-CPSs, but it requires a multidimensional search.

Inspired by the proposed LPWD in Refs. 21 and 22 and considering the tradeoff between the computational cost and the performance of parameter estimation, an effective parameter estimation algorithm for multi-CPSs is introduced based on the local polynomial ambiguity function (LPAF) in this paper, which does not suffer from the considerable cross-term interference and heavy computational burden due to its moderate order of nonlinearity, coherent integration via fast Fourier transform (FFT). Then, the high-quality ISAR image can be achieved by the corresponding ISAR imaging algorithm based on LPAF.

This paper is organized as follows. ISAR imaging model with complex motion is given in Sec. 2. In Sec. 3, the parameter estimation algorithm for CPS is proposed based on LPAF. In Sec. 4, the performance of LPAF is discussed in detail, which mainly includes the computational cost and the robustness in the presence of noise. The LPAF-based ISAR imaging algorithm is presented in Sec. 5. Finally, the application of the LPAF-based ISAR imaging algorithm and the conclusion are given in Secs. 6 and 7, respectively.

\section{Inverse Synthetic Aperture Radar Imaging Model with Complex Motion}

ISAR imaging model on the imaging project plane is shown in Fig. 1, where the radar is supposed to locate at the same plane with the target, and to orient along the $X$ axis. $P_{i}\left(x_{i}, y_{i}\right)$ is the location on the $i$ 'th point scatterer in the Cartesian coordinates with its origin at the geometric center $O$ of the target, and the $i$ 'th point scatterer can also be described as $P_{i}\left(r_{i}, \theta_{i}\right)$, where $x_{i}=r_{i} \cos \theta_{i}, y_{i}=r_{i} \sin \theta_{i} . R_{t}$ and $\theta\left(t_{m}\right)$ are the translational distance and rotational displacement, respectively.

The instant distance $R\left(t_{m}\right)$ between the radar and the $i$ 'th point scatterer at the instant slow time $t_{m}$ can be approximated as

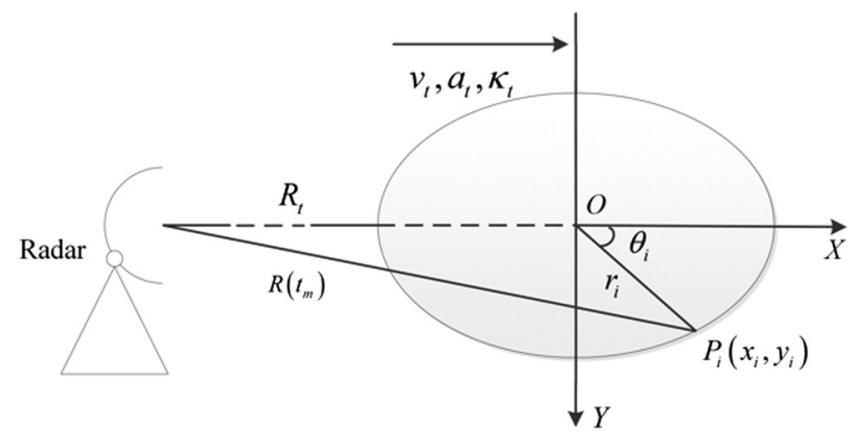

Fig. 1 ISAR imaging model on the project plane. 
Lv, Su, and Zheng: Inverse synthetic aperture radar imaging of targets with complex motion based...

$$
\begin{aligned}
R\left(t_{m}\right) & =\left\{R_{t}^{2}+r_{i}^{2}+2 R_{t} r_{i} \cos \left[\theta\left(t_{m}\right)+\theta_{i}\right]\right\}^{1 / 2} \\
& \approx R_{t}+\frac{r_{i}^{2}+2 R_{t} r_{i} \cos \left[\theta\left(t_{m}\right)+\theta_{i}\right]}{2 R_{t}} \\
& \approx R_{t}+r_{i} \cos \theta_{i} \cos \theta\left(t_{m}\right)-r_{i} \sin \theta_{i} \sin \theta\left(t_{m}\right) \\
& =R_{t}+x_{i} \cos \theta\left(t_{m}\right)-y_{i} \sin \theta\left(t_{m}\right) .
\end{aligned}
$$

For targets with complex motion, e.g., the highly maneuvering airplane and ship fluctuation with oceanic waves, $R_{t}$ and $\theta\left(t_{m}\right)$ are time-varying and can be expressed in Taylor series as follows:

$$
\begin{gathered}
R_{t}=R_{0}+v_{t} t_{m}+\frac{1}{2} a_{t} t_{m}^{2}+\frac{1}{6} \kappa_{t} t_{m}^{3} \cdots, \\
\theta\left(t_{m}\right)=\alpha t_{m}+\frac{1}{2} \beta t_{m}^{2}+\frac{1}{6} \gamma t_{m}^{3} \cdots,
\end{gathered}
$$

where $R_{0}$ is the initial distance of the geometric center to radar, and $v_{t}, a_{t}$, and $\kappa_{t}$ denote the radial velocity, acceleration, and acceleration rate of the target corresponding to the radar, respectively. Similarly, $\alpha, \beta$, and $\gamma$ denote rotational velocity, rotational acceleration, and rotational acceleration rate, respectively. For the maneuverability of the target, we can give a third-order approximation of $R_{t}$ and $\theta\left(t_{m}\right)$, and $\cos \theta\left(t_{m}\right)$ and $\sin \theta\left(t_{m}\right)$ can be approximated as 1 and $\theta\left(t_{m}\right)$, respectively. Therefore, substituting Eqs. (2) and (3) into Eq. (1), we can obtain

$$
R\left(t_{m}\right) \approx R_{0}+x_{i}+\left(v_{t}-y_{i} \alpha\right) t_{m}+\frac{1}{2}\left(a_{t}-y_{i} \beta\right) t_{m}^{2}+\frac{1}{6}\left(\kappa_{t}-y_{i} \gamma\right) t_{m}^{3}
$$

Assume the transmitted LFM signal takes the following form:

$$
s_{t}(\hat{t})=\operatorname{rect}\left(\frac{\hat{t}}{T}\right) \exp \left[j 2 \pi\left(f_{c} \hat{t}+\frac{1}{2} \mu \hat{t}^{2}\right)\right]
$$

where $\hat{t}, T, f_{c}$, and $\mu$ are the fast time, the pulsewidth, the carrier frequency, and the chirp rate (CR), respectively. Then, the baseband echo signal of the scatterer $P_{i}$ can be expressed as

$$
s_{r}\left(\hat{t}, t_{m}\right)=\sigma_{i} \operatorname{rect}\left[\frac{\hat{t}-2 R\left(t_{m}\right) / c}{T}\right] \exp \left(j 2 \pi\left\{-f_{c} \frac{2 R\left(t_{m}\right)}{c}+\frac{1}{2} \mu\left[\hat{t}-\frac{2 R\left(t_{m}\right)}{c}\right]^{2}\right\}\right),
$$

where $\sigma_{i}$ denotes the amplitude of the $i$ 'th point scatterer.

After the pulse compression by using the matched filter (MF) $H(\hat{t})=\operatorname{rect}(\hat{t} / T) \exp \left(j \pi \mu \hat{t}^{2}\right)$, we have

$$
s_{\mathrm{pc}}\left(\hat{t}, t_{m}\right)=\sigma_{i} B \operatorname{sinc}\left\{B\left[\hat{t}-\frac{2 R\left(t_{m}\right)}{c}\right]\right\} \exp \left[-j \frac{4 \pi}{\lambda} R\left(t_{m}\right)\right]
$$

where $B$ is the bandwidth of the transmitted signal.

In Eq. (7), the complex motion will result in range migration and Doppler frequency shift. The translational range migration has the same feature for all scatterers and has no contribution for ISAR imaging. Hence, in this paper, the standard range alignment method ${ }^{24}$ and the phase gradient autofocus method ${ }^{25}$ are utilized to eliminate the effect caused by translation, and keystone transform to correct the rotational range migration. Here, we only concentrate on the Doppler frequency shift caused by rotation.

Consequently, after the motion compensation (the range migration compensation and the translational induced phase error correction), the azimuth echo of the $i$ 'th scatter in the range-azimuth domain can be rewritten as 
$s_{\mathrm{pc}}\left(\hat{t}, t_{m}\right)=\sigma_{i} B \operatorname{sinc}\left\{B\left[\hat{t}-\frac{2\left(R_{o}+x_{i}\right)}{c}\right]\right\} \exp \left\{j 2 \pi\left[\left(\frac{2 y_{i} \alpha}{\lambda}\right) t_{m}+\frac{1}{2}\left(\frac{2 y_{i} \beta}{\lambda}\right) t_{m}^{2}+\frac{1}{6}\left(\frac{2 y_{i} \gamma}{\lambda}\right) t_{m}^{3}\right]\right\}$.

In realistic ISAR imaging applications, there will be multiple scatterers in a range bin simultaneously, which is more accurate and has been confirmed in many literatures. Thus, the azimuth echo signals in a range bin can be rewritten as

$$
s\left(t_{m}\right)=\sum_{i=1}^{Q} A_{i} \exp \left[j 2 \pi\left(\phi_{i, 1} t_{m}+\frac{1}{2} \phi_{i, 2} t_{m}^{2}+\frac{1}{6} \phi_{i, 3} t_{m}^{3}\right)\right]
$$

where $Q$ is the total number of scatterers in a certain range bin, and $A_{i}, \phi_{i, 1}=2 y_{i} \alpha / \lambda$, $\phi_{i, 2}=2 y_{i} \beta / \lambda$, and $\phi_{i, 3}=2 y_{i} \gamma / \lambda$ denote the amplitude, centroid frequency (CF), CR, and quadratic chirp rate (QCR) of the $i$ 'th point scatterer, respectively. We can see from Eq. (9) that it is the CR and the QCR that induce the Doppler frequency shift and have to be estimated accurately and then compensated for ISAR imaging.

\section{Parameters Estimation for Cubic Phase Signal}

\subsection{Introduction of Local Polynomial Wigner Distribution}

LPWD, ${ }^{20}$ a special kind of time-frequency distribution proposed by Stankovic, is widely used for the parameters estimation of multi-CPSs. Then the ISAR imaging algorithm ${ }^{21,22}$ is also proposed based on LPWD. LPWD is defined in Ref. 20 as follows:

$$
W(\vec{\omega}, t)=\int_{-\infty}^{+\infty} s\left(t+\frac{\tau}{2}\right) s^{*}\left(t-\frac{\tau}{2}\right) \exp [-j 2 \pi \theta(\vec{\omega}, \tau)] \mathrm{d} \tau,
$$

where $*$ and $\tau$ denote the complex conjugation and lag-time variable, respectively, and

$$
\theta(\vec{\omega}, \tau)=\omega_{1} \tau+\omega_{2} \frac{\tau^{2}}{2 !}+\omega_{3} \frac{\tau^{3}}{3 !}+\ldots+\omega_{m} \frac{\tau^{m}}{m !},
$$

where $\vec{\omega}=\left(\omega_{1}, \omega_{2}, \omega_{3} \ldots, \omega_{m}\right)$ is an $m$-dimensional variable space.

Thereby, the instantaneous frequency estimation of polynomial phase signal (PPS) can be achieved with a significantly reduced distribution dimension.

\subsection{Definition of Local Polynomial Ambiguity Function}

Considering an analytic signal $s(t)$, the ambiguity function (AF) is defined as

$$
A(\tau, \omega)=\int_{-\infty}^{+\infty} s\left(t+\frac{\tau}{2}\right) s^{*}\left(t+\frac{\tau}{2}\right) \exp (-j 2 \pi \omega t) \mathrm{d} t
$$

Borrowing the idea from the definitions of LPWD and AF, the LPAF is defined as follows:

$$
\operatorname{LPAF}(\vec{\omega}, \tau)=\int_{-\infty}^{+\infty} s\left(t+\frac{\tau}{2}\right) s^{*}\left(t-\frac{\tau}{2}\right) \exp [-j 2 \pi \theta(\vec{\omega}, t)] \mathrm{d} t .
$$

Similar to the definition of LPWD, we have

$$
\theta(\vec{\omega}, t)=\omega_{1} t+\omega_{2} \frac{t^{2}}{2 !}+\omega_{3} \frac{t^{3}}{3 !}+\ldots+\omega_{m} \frac{t^{m}}{m !},
$$

where $\vec{\omega}=\left(\omega_{1}, \omega_{2}, \omega_{3} \ldots, \omega_{m}\right)$ is an $m$-dimensional variable space. 
Consider mono-CPS with the following form:

$$
s(t)=A \exp \left[j 2 \pi\left(\phi_{1} t+\frac{1}{2} \phi_{2} t^{2}+\frac{1}{6} \phi_{3} t^{3}\right)\right]
$$

where $A, \phi_{1}, \phi_{2}$, and $\phi_{3}$ denote the amplitude, CF, CR, and QCR, respectively. LPAF for $s(t)$ can be expressed as

$$
\operatorname{LPAF}\left(\omega_{1}, \omega_{2}, \tau\right)=\int_{-\infty}^{+\infty} s\left(t+\frac{\tau}{2}\right) s^{*}\left(t-\frac{\tau}{2}\right) \exp \left[-j 2 \pi\left(\omega_{1} t+\omega_{2} \frac{t^{2}}{2}\right)\right] \mathrm{d} t
$$

Substituting Eq. (15) in Eq. (16), we obtain

$$
\operatorname{LPAF}\left(\omega_{1}, \omega_{2}, \tau\right)=A^{2} \exp [j \Phi(\tau)] \int_{-\infty}^{+\infty} \exp \left[-j 2 \pi\left(\omega_{1}-\phi_{2} \tau\right) t\right] \exp \left[-j 2 \pi\left(\frac{1}{2} \omega_{2}-\frac{1}{2} \phi_{3} \tau\right) t^{2}\right] \mathrm{d} t
$$

where $\Phi(\tau)=2 \pi\left(\phi_{1} \tau+\phi_{3} \tau^{3} / 24\right)$ is independent of variable $t$. Similar to the LPWD in Ref. 21, we choose the special lag-time instance $\tau=\tau_{0}$ to achieve the LPAF; then, Eq. (17) can be simplified as

$$
\operatorname{LPAF}\left(\omega_{1}, \omega_{2}\right)=D^{2} \int_{-\infty}^{+\infty} \exp \left[-j 2 \pi\left(\omega_{1}-\phi_{2} \tau_{0}\right) t\right] \exp \left[-j 2 \pi\left(\frac{1}{2} \omega_{2}-\frac{1}{2} \phi_{3} \tau_{0}\right) t^{2}\right] \mathrm{d} t
$$

where $D$ is the relative amplitude. It is worthwhile noting that a sole peak appears in the $\omega_{1}-\omega_{2}$ plane, that is, the chirp rate-quadratic chirp rate distribution (CRQCRD) of $\left|\operatorname{LPAF}\left(\omega_{1}, \omega_{2}\right)\right|$ gets the maximum in the following case:

$$
\left\{\begin{array}{l}
\omega_{1}=\phi_{2} \tau_{0} \\
\omega_{2}=\phi_{3} \tau_{0}
\end{array} .\right.
$$

From Eq. (19), it can be seen that the different lag-time instance $\tau_{0}$ indicates the different zoom factor, which can enlarge the estimation ranges of CR and QCR to avoid the spectrum aliasing and estimation error ${ }^{26}$ in some extreme ISAR imaging case, e.g., higher maneuverability. On the other hand, the symmetric instantaneous self-correlation operation will shorten the effective signal in Eq. (16) when $\tau_{0} \neq 0$. Based on the recommendation from Ref. 27, the optimal discrete lag is $N_{\tau_{0} / 2}^{\mathrm{opt}}=(N+1) / 2 H$, where $H$ is the PPS order. For convenience to analyze, we choose $\tau_{0}=1$ so that the values of CR and QCR can be read directly from CRQCRD.

Thereby, we can estimate the parameters $\phi_{2}$ and $\phi_{3}$ simultaneously by searching the position of the maximum of $\left|\operatorname{LPAF}\left(\omega_{1}, \omega_{2}\right)\right|$.

$$
\left(\hat{\phi}_{2}, \hat{\phi}_{3}\right)=\underset{\omega_{1}, \omega_{2}}{\arg \max }\left|\operatorname{LPAF}\left(\omega_{1}, \omega_{2}\right)\right|
$$

With the estimated parameters $\left(\hat{\phi}_{2}, \hat{\phi}_{3}\right)$ and dechirp technique, the other parameters can be estimated via FFT after compensating the high-order phase terms.

According to the aforementioned analyses on LPWD and LPAF for CPS, we know that LPAF is similar to LPWD except for the definition of $\theta(\vec{\omega}, \Delta)$ in Eqs. (11) and (14), where $\Delta=\tau$ for LPWD and $\Delta=t$ for LPAF. However, instead of the special time instance as $t=0,{ }^{21}$ LPAF can choose the different lag-time instance $\tau_{0}$ to introduce a zoom factor adjusting the estimation ranges of CR and QCR in Eq. (19). It must be emphasized that such a difference circumvents the problems of LPWD including spectrum aliasing, error propagation, and computational burden, which will be discussed in Sec. 4. 


\subsection{Local Polynomial Ambiguity Function for Multicomponent Cubic Phase Signals}

In this section, the performance of LPAF for multi-CPSs is shown. As we know, correlation operation will result in the cross-term effects among multi-CPSs, which determines whether the proposed algorithm is suitable for multi-CPSs or not. For simplicity, we choose special case as $\tau_{0}=1$ and consider two-component CPSs in the following structure:

$$
\begin{aligned}
s(t) & =s_{p}(t)+s_{q}(t) \\
& =A_{p} \exp \left[j 2 \pi\left(\phi_{p, 1} t+\phi_{p, 2} t^{2}+\phi_{p, 3} t^{3}\right)\right]+A_{q} \exp \left[j 2 \pi\left(\phi_{q, 1} t+\phi_{q, 2} t^{2}+\phi_{q, 3} t^{3}\right)\right] .
\end{aligned}
$$

Substituting Eq. (21) in Eq. (16), we obtain the LPAF for $s(t)$ in Eq. (21) as follows:

$$
\begin{aligned}
\operatorname{LPAF}\left(\omega_{1}, \omega_{2}\right)= & D_{p}^{2} \int_{-\infty}^{+\infty} \exp \left[-j 2 \pi\left(\omega_{1}-\phi_{p, 2}\right) t\right] \times \exp \left[-j 2 \pi\left(\frac{1}{2} \omega_{2}-\frac{1}{2} \phi_{p, 3}\right) t^{2}\right] \mathrm{d} t \\
& +D_{q}^{2} \int_{-\infty}^{+\infty} \exp \left[-j 2 \pi\left(\omega_{1}-\phi_{q, 2}\right) t\right] \times \exp \left[-j 2 \pi\left(\frac{1}{2} \omega_{2}-\frac{1}{2} \phi_{q, 3}\right) t^{2}\right] \mathrm{d} t \\
& +\operatorname{LPAF}_{p q, \text { cross }}\left(\omega_{1}, \omega_{2}\right)+\operatorname{LPAF}_{q p, \text { cross }}\left(\omega_{1}, \omega_{2}\right) .
\end{aligned}
$$

Note that we have two cross-terms $\operatorname{LPAF}_{p q \text {, cross }}\left(\omega_{1}, \omega_{2}\right)$ and $\operatorname{LPAF}_{q p \text {,cross }}\left(\omega_{1}, \omega_{2}\right)$, and we only take $\operatorname{LPAF}_{p q \text {,cross }}\left(\omega_{1}, \omega_{2}\right)$ as an example to analyze. Obviously, $\operatorname{LPAF}_{p q \text {,cross }}\left(\omega_{1}, \omega_{2}\right)$ is with the form

$\operatorname{LPAF}_{p q, \operatorname{cross}}\left(\omega_{1}, \omega_{2}\right)$

$$
\begin{aligned}
= & D_{p q, \mathrm{cross}} \int_{-\infty}^{+\infty} \exp \left\{-j 2 \pi\left[\omega_{1}-\left(\phi_{p, 1}-\phi_{q, 1}\right)-\frac{1}{2}\left(\phi_{p, 2}+\phi_{q, 2}\right)-\frac{1}{8}\left(\phi_{p, 3}-\phi_{q, 3}\right)\right] t\right\} \\
& \times \exp \left\{-j 2 \pi\left[\omega_{2}-\frac{1}{2}\left(\phi_{p, 2}-\phi_{q, 2}\right)-\frac{1}{4}\left(\phi_{p, 3}+\phi_{q, 3}\right)\right] t^{2}\right\} \\
& \times \exp \left\{j 2 \pi\left[\frac{1}{6}\left(\phi_{p, 3}-\phi_{q, 3}\right)\right] t^{3}\right\} \mathrm{d} t
\end{aligned}
$$

where $D_{p q \text {,cross }}$ is irrelevant to the variable $t$.

As is obvious from Eq. (23), when $\phi_{p, 3} \neq \phi_{q, 3}$, the auto-terms can be detected correctly by peak detection, and the phase coefficients can be estimated easily. Here, assume that the received signal contains the same QCR, i.e., $\phi_{p, 3}=\phi_{q, 3}$. Then, $\operatorname{LPAF}_{p q, \text { cross }}\left(\omega_{1}, \omega_{2}\right)$ can be further simplified as

$$
\begin{aligned}
& \mathrm{LPAF}_{p q, \text { cross }}\left(\omega_{1}, \omega_{2}\right)=D_{p q, \text { cross }} \int_{-\infty}^{+\infty} \exp \left\{-j 2 \pi\left[\omega_{1}-\left(\phi_{p, 1}-\phi_{q, 1}\right)-\frac{1}{2}\left(\phi_{p, 2}+\phi_{q, 2}\right)\right] t\right\} \\
& \quad \times \exp \left\{-j 2 \pi\left[\omega_{2}-\frac{1}{2}\left(\phi_{p, 2}-\phi_{q, 2}\right)-\frac{1}{2}\left(\phi_{p, 3}\right)\right] t^{2}\right\} \mathrm{d} t .
\end{aligned}
$$

From Eq. (24), we can find that the spurious peak will be located at

$$
\left\{\begin{array}{l}
\omega_{1}=\left(\phi_{p, 1}-\phi_{q, 1}\right)+\frac{1}{2}\left(\phi_{p, 2}+\phi_{q, 2}\right) \\
\omega_{2}=\frac{1}{2}\left(\phi_{p, 2}-\phi_{q, 2}\right)+\frac{1}{2}\left(\phi_{p, 3}\right)
\end{array} .\right.
$$

It is worthwhile to mention that only when $\phi_{p, 3}=\phi_{q, 3}$, the LPAF for $s(t)$ causes spurious peak, which affects the detection and estimation for multi-CPSs. Fortunately, the phase coefficients $\phi_{j}(j=0,1,2 \ldots)$ are not only related to target rotational parameter (e.g., CF corresponding to the initial rotational velocity, $\mathrm{CR}$ corresponding to the rotational acceleration, and QCR corresponding to the rotational acceleration rate), but also proximately proportional to the cross-range coordinates. Thus, for different scatterers with different coordinates in a range bin, 
Table 1 Parameters for CPS.

\begin{tabular}{lcccr}
\hline \hline & $A_{i}$ & $\phi_{i, 1}$ & $\phi_{i, 2}$ & $\phi_{i, 3}$ \\
\hline$i=1$ & 1 & $40 \mathrm{~Hz}$ & $30 \mathrm{~Hz} / \mathrm{s}$ & $-60 \mathrm{~Hz} / \mathrm{s}^{2}$ \\
$i=2$ & 1.05 & $-30 \mathrm{~Hz}$ & $-80 \mathrm{~Hz} / \mathrm{s}$ & $40 \mathrm{~Hz} / \mathrm{s}^{2}$ \\
\hline \hline
\end{tabular}

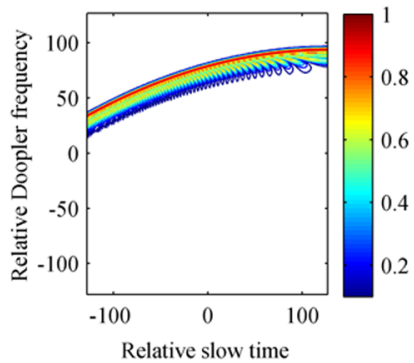

(a)

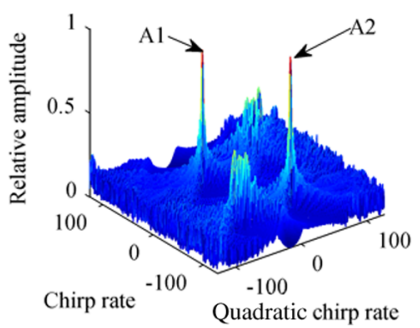

(d)

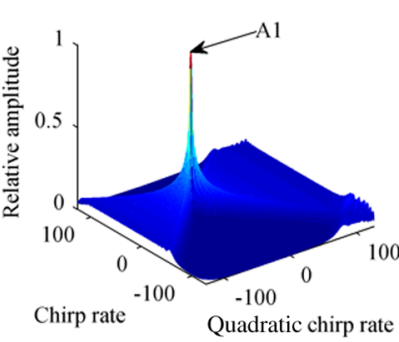

(b)

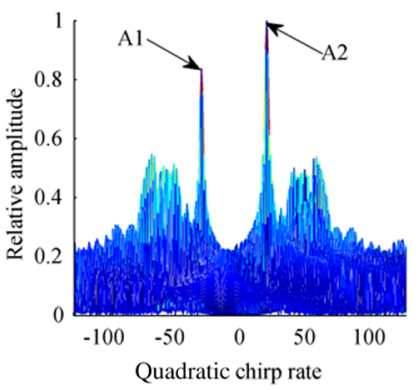

(e)

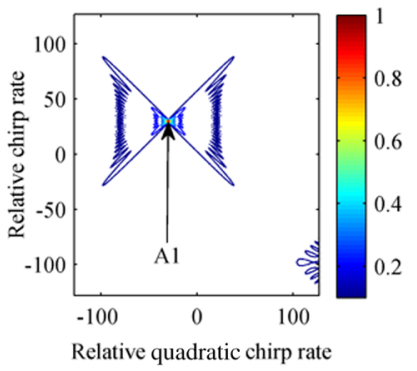

(c)

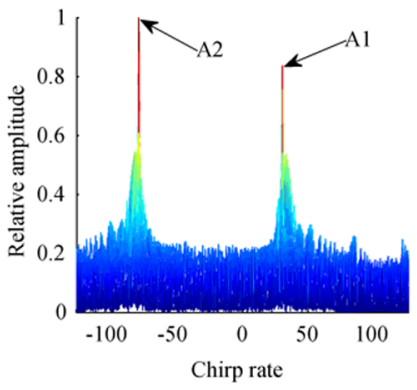

(f)

Fig. 2 Simulation results of example 1: (a) Wigner-Ville distribution of first CPS, (b) stereogram of CRQCRD in first CPS, (c) contour of (b), (d) stereogram of CRQCRD in two CPSs, (e) $X Z$ plane of (d), and (f) $Y Z$ plane of (d).

we have $\phi_{p 3} \neq \phi_{q 3}(p \neq q) \cdot{ }^{14}$ Accordingly, the cross-terms of the LPAF induced by multi-CPSs can be avoided effectively.

Example 1. Consider a mono-component CPS and two-component CPSs, respectively. The sampling frequency and the effective length of signal are $256 \mathrm{~Hz}$ and 256, respectively. The signal parameters are shown in Table 1.

The WVD of CPS-A1 is shown in Fig. 2(a), in which the curves appear due to QCR of CPS. With the proposed parameter estimation algorithm, a sole peak appears in the CRQCRD in Figs. 2(b) and 2(c), where CR and QCR can be obtained by peak detection. Moreover, we obtain the CRQCRD of the two-component CPSs in Fig. 2(d). For easy visualization, the stereogram, the right view ( $X Z$ plane), and the left view ( $X Z$ plane) of CRQCRD are depicted in Figs. 2(d)2(f), where two different peaks appear in CRQCRD. Therefore, the corresponding parameters of each component can be easily estimated via searching the peaks and CLEAN technique, ${ }^{7,8}$ sequentially.

\section{Performance of Local Polynomial Ambiguity Function}

In this section, we will discuss the performance of LPAF, mainly including the computational cost and the robustness in the presence of noise, which determine whether the proposed algorithm can be utilized for the parameter estimation of multi-CPSs in realistic ISAR imaging applications or not. In order to verify the advantages of the proposed estimation algorithm, other two representative algorithms, MCPF and LPWD, are chosen as reference to compare with the proposal. 


\subsection{Analysis of the Computational Cost}

For MCPF, the estimation procedures of the parameter $\phi_{3}$ require $O\left(3 N_{t_{m}}^{2}\right)$ complex multiply operations (CMs) for definition of multilinear function and $O\left(N_{t_{m}}^{3}\right)$ operations for discrete Fourier transform (DFT) along the nonuniformly spaced lag-time axis. For estimation of the parameter $\phi_{2}$, the implementation procedures require $O\left(N_{t_{m}}^{2}\right)$ operations for CPF and $O\left(N_{t_{m}}^{3}\right)$ operations for DFT due to the two-dimensional (2-D) kernel. Thus, the computational complexity with MCPF is in the order of $O\left(N_{t_{m}}^{3}\right)$. According to Refs. 11 and 13, the nonuniform fast Fourier transform (NUFFT) ${ }^{11,13}$ can be utilized to speed up the implementation of the Fourier transform along the nonuniformly spaced lag-time axis. Unfortunately, the interpolation necessary for NUFFT is not preferable in some realistic applications.

The LPAF algorithm, which can directly obtain CRQCRD, estimates CR and QCR with 2-D maximizations and can reduce error propagation effect to some extent. According to the aforementioned analyses and discussion on the modified discrete chirp-Fourier transform, ${ }^{28,29}$ which retains capability of efficient FFT implementation, the implementation procedures of parameters estimation based on LPAF include (1) the self-correlation operation in Eq. (16), after which the signal has the form of the linear frequency modulated signal, (2) FFT operation after matching the QCR $\phi_{3}$ in Eq. (18), and (3) the parameters estimation by peak detection technique. In this paper, the searching range of the parameter $\phi_{3}$ is $\left[-\operatorname{ROUND}\left(F t_{m} / 2\right), \operatorname{ROUND}\left(F t_{m} / 2\right)\right]$, where $\operatorname{ROUND}(\cdot)$ and $F t_{m}$ denote the round-up operator and pulse repetition frequency (PRF), respectively. The resolution of the parameter $\phi_{3}$ is PRF $/ N_{t_{m}}$, where $N_{t_{m}}$ is the effective length of $t_{m}$ (the same as the number of effective echoes); thus, the searching point of the parameter $\phi_{3}$ is exactly $N_{t_{m}}$ in ISAR imaging. In conclusion, the computational complexity with this approach is about in the order of $O\left(N_{t_{m}}^{2} \log _{2} N_{t_{m}}\right)$.

For LPWD, the implementation procedures for the estimation of parameters $\phi_{1}$ and $\phi_{3}$ are analogous to the LPAF algorithm. However, the parameter $\phi_{2}$ is estimated by the integrated form of cubic phase function, which includes the defined bilinear function $\left[O\left(N_{t_{m}}^{2}\right)\right]$ and Fourier transform $\left[O\left(N_{t_{m}}^{3}\right)\right]$ along the nonuniformly spaced lag-time axis. Therefore, the computational complexity with the LPWD-based algorithm is also in the order of $O\left(N_{t_{m}}^{3}\right)$, which is obviously greater than the LPAF algorithm.

It is obvious that, compared with MCPF and LPWD, the proposed algorithm is computationally more efficient than the other two algorithms since the latter involves nonuniformly spaced data computation. Furthermore, as mentioned in Ref. 21, MCPF estimates phase coefficients one by one via dechirp technique, which will reduce the estimation precision due to error propagation. On the contrary, the LPAF algorithm can obtain CR and QCR simultaneously. Table 2 gives the computational costs of the three algorithms.

\subsection{Analysis of Performance in Noise}

In order to further illustrate the practicability of the proposed parameter estimation algorithm, the robustness of performance against noise, which is evaluated by input-output signal-to-noise ratio $(\mathrm{SNR})^{9}$ and mean square error (MSE), ${ }^{30,31}$ will be analyzed in this section.

Example 2. Consider a mono-CPS as shown in Eq. (15); the sampling frequency and the effective length of signal are $256 \mathrm{~Hz}$ and 256 , respectively. The signal parameters are set as follows: $A=1, \phi_{1}=40 \mathrm{~Hz}, \phi_{2}=24 \mathrm{~Hz} / \mathrm{s}, \phi_{3}=60 \mathrm{~Hz} / \mathrm{s}^{2}$. Hereon, the CPS is embedded in complex white Gaussian noise, and the value of $\mathrm{SNR}_{\text {in }}$ varies from -5 to $2 \mathrm{~dB}$ in steps of $1 \mathrm{~dB}$, and 100 Monte Carlo simulations are performed for each $\mathrm{SNR}_{\text {in }}$ value. Meanwhile, the MSEs for the parameters $\phi_{2}$ and $\phi_{3}$ with the corresponding Cramer-Rao bounds (CRBs) ${ }^{30}$ are also obtained and shown in Figs. 3(b) and 3(c), respectively.

Table 2 Computational cost.

\begin{tabular}{llll}
\hline \hline Computation cost & LPWD & MCPF & LPAF \\
\hline Number of CMs & $O\left(N_{t_{m}}^{3}\right)$ & $O\left(N_{t_{m}}^{3}\right)$ & $O\left(N_{t_{m}}^{2} \log _{2} N_{t_{m}}\right)$ \\
\hline \hline
\end{tabular}




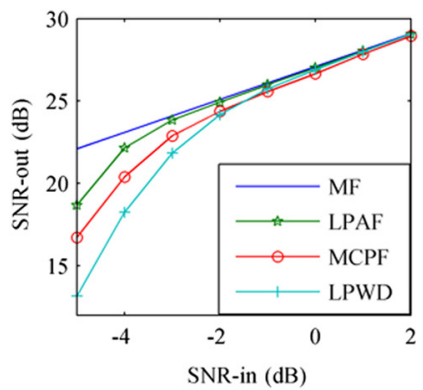

(a)

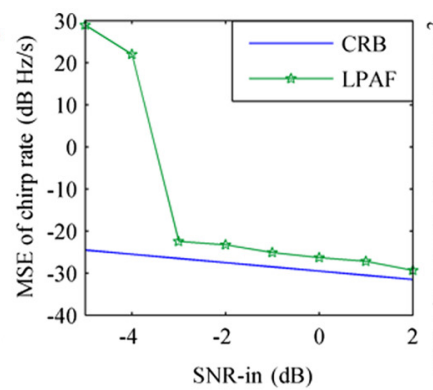

(b)

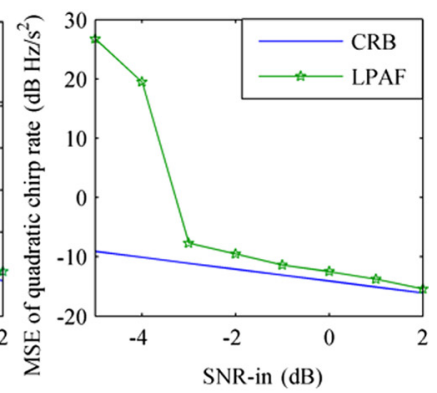

(c)

Fig. 3 Robustness of performance against noise: (a) input-output SNR, (b) MSE of CR estimation, and (c) MSE of QCR estimation.

In Fig. 3(a), MF, LPWD, and MCPF are chosen to compare with the proposed LPAF. For $\mathrm{MCPF}$, its implementation is similar to $\mathrm{PGCPF},{ }^{18}$ which defines a multilinear function of fourth order and estimates unknown parameters one by one with two one-dimensional maximizations. Thus, the performance of MCPF closely matches that of MF until the input SNR falls below $0 \mathrm{~dB}$, and the evidence for MCPF can be found in Ref. 10. For LPWD, its performance also closely matches that of MF until the input SNR falls below $0 \mathrm{~dB}$, and we can find the corresponding evidence in Ref. 21. According to the analyses in Refs. 8 and 11, compared to the self-correlation function $\left[s(\tau / 2) s^{*}(-\tau / 2)\right.$, for $\left.t=0\right]$ of LPWD, the self-correlation function $\left[s\left(t+\tau_{0} / 2\right) s^{*}\left(t-\tau_{0} / 2\right)\right.$, for $\left.\tau=\tau_{0}\right]$ of LPAF can reduce the influence of nonlinearity and benefit the performance in the presence of noise. Thereby, the proposed LPAF can obtain robust performance against noise and its threshold SNR is $-3 \mathrm{~dB}$. In addition, the MSEs for the estimations $\phi_{2}$ and $\phi_{3}$ are plotted in Figs. 3(b) and 3(c) as a function of SNR, respectively. Obviously, the MSEs of $\mathrm{CR}$ and QCR are close to the corresponding CRB when $\mathrm{SNR}_{\text {in }} \geq-3 \mathrm{~dB}$, which conforms to the result in Fig. 3(a).

\section{Inverse Synthetic Aperture Radar Imaging Algorithm Based on Local Polynomial Ambiguity Function}

On the above analyses and confirmation, a new ISAR imaging algorithm for target with complex motion is proposed based on LPAF. Detail implementation procedures are given as follows:

Step 1: Complete the range compression and motion compensation, and obtain data $s^{k}\left(t_{m}\right)$ of the $k^{\prime}$ th (where $1 \leq k \leq K$, and $K$ is the total number of range bins) range bin.

Step 2: Substitute $s^{k}\left(t_{m}\right)$ in Eq. (16).

$$
\begin{aligned}
\operatorname{LPAF}^{k}\left(\omega_{1}, \omega_{2}\right)= & \sum_{i=1}^{Q} D_{i}^{2} \int_{-\infty}^{+\infty} \exp \left[-j 2 \pi\left(\omega_{1}-\phi_{i, 2}\right) t\right] \exp \left[-j 2 \pi\left(\frac{1}{2} \omega_{2}-\frac{1}{2} \phi_{i, 3}\right) t^{2}\right] \mathrm{d} t \\
& +\operatorname{LPAF}_{\text {cross }}^{k}\left(\omega_{1}, \omega_{2}\right)+\operatorname{LPAF}_{\text {noise }}^{k}\left(\omega_{1}, \omega_{2}\right)
\end{aligned}
$$

where $\operatorname{LPAF}_{\text {cross }}^{k}\left(\omega_{1}, \omega_{2}\right)$ and $\operatorname{LPAF}_{\text {noise }}^{k}\left(\omega_{1}, \omega_{2}\right)$ denote the cross-terms and noise, respectively.

Step 3: Complete estimation of the parameters $\phi_{i, 2}$ and $\phi_{i, 3}$ of the $i$ 'th CPS by finding the location of peak.

$$
\left(\hat{\phi}_{i, 2}=\omega_{1}, \hat{\phi}_{i, 3}=\omega_{2}\right)=\underset{\omega_{1}, \omega_{2}}{\arg \max }\left|\operatorname{LPAF}^{k}\left(\omega_{1}, \omega_{2}\right)\right| .
$$

Step 4: Dechirp $s^{k}\left(t_{m}\right)$ with the estimated parameters $\phi_{i, 2}$ and $\phi_{i, 3}$ to estimate $\phi_{i, 1}$ and $A_{i}$.

$$
\left(\hat{A}_{i}=\frac{G}{N}, \hat{\phi}_{i, 1}=f\right)=\underset{G, f}{\arg \max }\left|\mathrm{FFT}\left\{s^{k}\left(t_{m}\right) \times \exp \left[-j 2 \pi\left(\frac{1}{2} \hat{\phi}_{i, 2} t_{m}^{2}+\frac{1}{6} \hat{\phi}_{i, 3} t_{m}^{3}\right)\right]\right\}\right| .
$$


Step 5: Subtract the estimated $i^{\prime}$ th CPS from the original signal $s^{k}\left(t_{m}\right)$ in frequency domain.

$$
\begin{aligned}
s^{k}\left(t_{m}\right)= & \operatorname{IFFT}\left(\operatorname{WIN}\left(\hat{\phi}_{i, 1}\right) \times \mathrm{FFT}\left\{s^{k}\left(t_{m}\right) \exp \left[-j 2 \pi\left(\frac{1}{2} \hat{\phi}_{i, 2} t_{m}^{2}+\frac{1}{6} \hat{\phi}_{i, 3} t_{m}^{3}\right)\right]\right\}\right) \\
& \times \exp \left[j 2 \pi\left(\frac{1}{2} \hat{\phi}_{i, 2} t_{m}^{2}+\frac{1}{6} \hat{\phi}_{i, 3} t_{m}^{3}\right)\right],
\end{aligned}
$$

where $\operatorname{WIN}\left(\hat{\phi}_{i, 1}\right)=\left\{\begin{array}{ll}0, & f-\Delta f / 2<\hat{\phi}_{i, 1}<f+\Delta f / 2 \\ 1, & \text { otherwise }\end{array}\right.$ is a notch filter centred at the CF $\hat{\phi}_{i, 1}$, and $\Delta f$ is the corresponding bandwidth.

Step 6: Renew $i=i+1$ and repeat steps 2 to 5 until the residual energy of the signal is less than the threshold $T$.

Step 7: Renew $k=k+1$ and repeat steps 1 to 6 until $k=K$.

\section{Inverse Synthetic Aperture Radar Imaging Results Based on Local Polynomial Ambiguity Function}

In this section, we will utilize the synthetic ship target and aircraft target with the model shown in Figs. 4(a) and 6(a) to verify the proposed ISAR imaging algorithm successively. Motion parameters and corresponding radar parameters are listed in Tables 3 and 4, respectively.

\subsection{Ship Target}

Similar to Refs. 10 and 13, a fluctuating ship target modeled as a set of ideal scatterers is provided to demonstrate the effectiveness of the proposed algorithm in this paper. After pulse compression and motion compensation, the received signal can be simplified as the 2-D reflectivity function shown in Eq. (8). Figure 4(b) gives the conventional RD imaging results with FFT operation along the azimuth slow time axis under the situation of $\mathrm{SNR}_{\mathrm{in}}=-3 \mathrm{~dB}$, which is

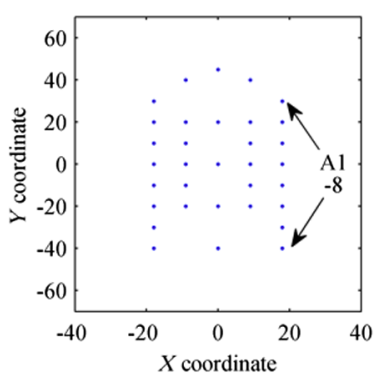

(a)

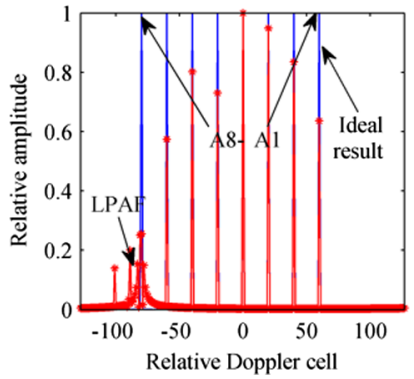

(d)

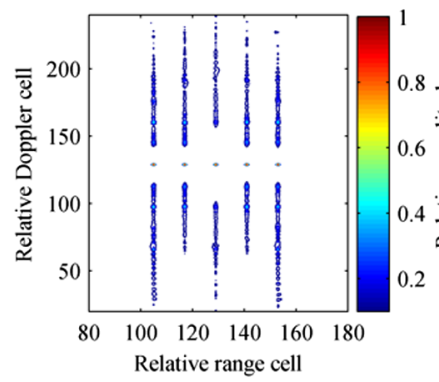

(b)

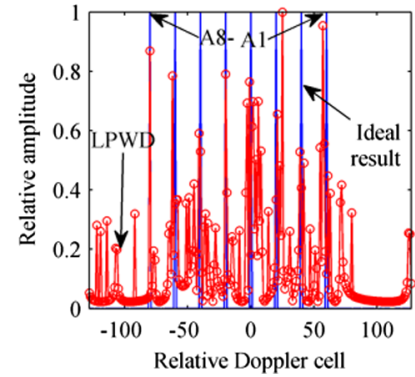

(e)

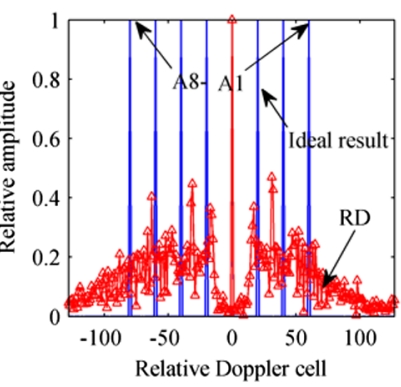

(c)

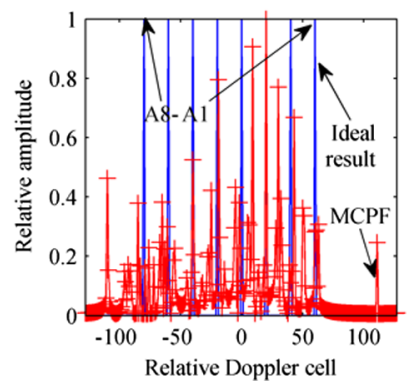

(f)

Fig. 4 (a) Ship target model, (b) result of the conventional RD algorithm, (c) result after the RD algorithm, (d) result after the Doppler spread compensation with LPAF, (e) result after the Doppler spread compensation with LPWD, and (f) result after the Doppler spread compensation with MCPF. 
Lv, Su, and Zheng: Inverse synthetic aperture radar imaging of targets with complex motion based...

Table 3 Simulation parameters of ship.

\begin{tabular}{lclcc}
\hline \multicolumn{1}{c}{ Radar } & & \multicolumn{3}{c}{ Ship target } \\
\hline Carrier frequency & $15 \mathrm{GHz}$ & Target distance & $24 \mathrm{~km}$ \\
Bandwidth & $200 \mathrm{MHz}$ & Motions & Translation & Rotation \\
Pulse repetition frequency (PRF) & $256 \mathrm{~Hz}$ & Velocity & $30 \mathrm{~m} / \mathrm{s}$ & $0.012 \mathrm{rad} / \mathrm{s}$ \\
Effective echo & 256 & Acceleration & $3 \mathrm{~m} / \mathrm{s}^{2}$ & $0.02 \mathrm{rad} / \mathrm{s}^{2}$ \\
Sample frequency & $200 \mathrm{MHz}$ & Acceleration rate & $2 \mathrm{~m} / \mathrm{s}^{3}$ & $0.04 \mathrm{rad} / \mathrm{s}^{3}$ \\
\hline \hline
\end{tabular}

Table 4 Simulation parameters of aircraft.

\begin{tabular}{lcccc}
\hline \multicolumn{1}{c}{ Radar } & & \multicolumn{2}{c}{ Aircraft target } \\
\hline Carrier frequency & $10 \mathrm{GHz}$ & Target distance & $40 \mathrm{~km}$ & \\
Bandwidth & $150 \mathrm{MHz}$ & Motions & Translation & Rotation \\
PRF & $256 \mathrm{~Hz}$ & Velocity & $20 \mathrm{~m} / \mathrm{s}$ & $0.03 \mathrm{rad} / \mathrm{s}$ \\
Echo number & 512 & Acceleration & $1 \mathrm{~m} / \mathrm{s}^{2}$ & $0.04 \mathrm{rad} / \mathrm{s}^{2}$ \\
Sample frequency & $300 \mathrm{MHz}$ & Acceleration rate & $2 \mathrm{~m} / \mathrm{s}^{3}$ & $0.08 \mathrm{rad} / \mathrm{s}^{3}$ \\
\hline \hline
\end{tabular}

blurred severely by CR and QCR. In this section, we choose the special lag-time instance $\tau_{0}=1$ to achieve the LPAF so that CR and QCR can be read directly.

To demonstrate the accuracy of the parameters estimation by the proposed LPAF, the data of the 153rd range bin in Fig. 4(b) are extracted to analyze, and the corresponding CRs and QCRs of these scatterers are estimated as shown in Table 5. Then, with the estimated CRs and QCRs, we compensate the Doppler frequency shift and obtain Figs. 4(d)-4(f), where the ideal matched results are also shown to make a comparison with each mentioned algorithm. Compared with the results in Figs. 4(c), 4(e), and 4(f), the result obtained by LPAF in Fig. 4(d) is very close to the ideal results, which demonstrate that the accuracy of the parameters estimation by the proposed LPAF is high. Although some spurious peaks appear in Fig. 4(d), the same as in Table 5, the LPAF is also better than other algorithms in the estimation accuracy and suppression on cross-terms.

In addition, the comparison of the LPAF-based algorithm with the MCPF-based algorithm and the LPWD-based algorithm for ISAR imaging is discussed. Generally, to quantitatively evaluate the performance of the proposed algorithm, the MSEs of 2-D reconstructed coordinates are defined as MSE $=\left\|\Re_{\text {est }}-\Re\right\|_{2}$, where $\Re$ and $\Re_{\text {est }}$ represent the original data and the reconstructed data obtained by the proposed algorithm, that is, the corresponding relation between $\Re$ and $\Re_{\text {est }}$ is determinate and known ahead of time. However, the number and the position of the scatterers on target are usually unknown and cannot be obtained in advance. Entropy can be used

Table 5 Results of parameters estimation.

\begin{tabular}{|c|c|c|c|c|c|c|c|c|c|c|}
\hline Signal parameters & $\mathrm{A} 1$ & $\mathrm{~A} 2$ & A3 & A4 & A5 & A6 & A7 & A8 & \multicolumn{2}{|c|}{$\begin{array}{l}\text { Spurious } \\
\text { peak }\end{array}$} \\
\hline $\mathrm{CR}(\mathrm{Hz} / \mathrm{s})$ & 60 & 40 & 20 & 0 & -20 & -40 & -60 & -80 & & \\
\hline Estimated CR $(\mathrm{Hz} / \mathrm{s})$ & 60 & 40 & 20 & 0 & -20 & -40 & -61 & -75 & -54 & -42 \\
\hline QCR $\left(\mathrm{Hz} / \mathrm{s}^{2}\right)$ & 120 & 80 & 40 & 0 & -40 & -80 & -120 & -160 & & \\
\hline Estimated QCR $\left(\mathrm{Hz} / \mathrm{s}^{2}\right)$ & 120 & 80 & 40 & 0 & -38 & -80 & -124 & -84 & -38 & 82 \\
\hline
\end{tabular}


Table 6 Entropies and time of ISAR images in Fig. 5.

\begin{tabular}{lccc}
\hline \hline & Fig. 5(a) & Fig. 5(b) & Fig. 5(c) \\
\hline Entropies & 3.4421 & 4.8379 & 4.2688 \\
Simulation time (s) & 1.2318 & 110.0910 & 202.9427 \\
\hline \hline
\end{tabular}

to measure the smoothness of a distribution function. The smoother a distribution function, the larger is its entropy. ${ }^{10,13}$ Owing to this property, we use the entropy of Eq. (30) as a criterion to measure the quality of the image in Table 6. Better focus results in a sharper image and thus smaller entropy. Meanwhile, simulation times of Fig. 5 (only including effective range bins) are also listed to certify the high efficiency of LPAF-based ISAR imaging algorithm.

$$
E=\sum_{m} \sum_{n} \frac{|I(m, n)|^{2}}{S} \operatorname{In} \frac{S}{|I(m, n)|^{2}},
$$

where $S=\sum_{m} \sum_{n}|I(m, n)|^{2}$.

From Fig. 5, we see that LPAF-based ISAR imaging algorithm has better suppression on cross-terms compared with the LPWD-based ISAR imaging algorithm and MCPF-based ISAR imaging algorithm. Evidently, most scatterers are relocated correctly in Fig. 5(a). Due to the four order of nonlinearity of MCPF and error propagation effect of LPWD (the estimation precision for CR is influenced by QCR), the spurious scatterers in Figs. 5(b) and 5(c) are more than those in Fig. 5(a). Furthermore, the entropy of Fig. 5(a) is also smaller than those of Figs. 5(b) and 5(c) in Table 6, which further shows that the LPAF-based ISAR imaging algorithm can obtain a well-focused image.

\subsection{Aircraft Target}

In this section, the synthetic aircraft target as shown in Fig. 6(a), which consists of 141 scatterers, is chosen to demonstrate the validity of the proposed ISAR imaging algorithm in this paper. We know that the CR and QCR of the azimuth echo in ISAR imaging are determined by the target's size and the effective rotating vector. When the target's size is a little larger or its maneuverability is a little higher, we have to adjust the parameter estimation range to meet the practical requirements. Here, we choose the special instance $\tau_{0}=1 / 2$ to enlarge the parameter estimation ranges and avoid spectrum aliasing and estimation error.

Obviously, the image obtained by the traditional RD algorithm is blurred severely in Fig. 6(b). In order to confirm the validity of the proposed parameter estimation algorithm, when all scatterers have been corrected into right slant range bins after motion compensation, we extract the data of the 211 st range bin to analyze in detail. Under the situation of $\mathrm{SNR}_{\mathrm{in}}=4 \mathrm{~dB}$, the WVD as shown in Fig. 6(c) is computed for the radar echo signal of

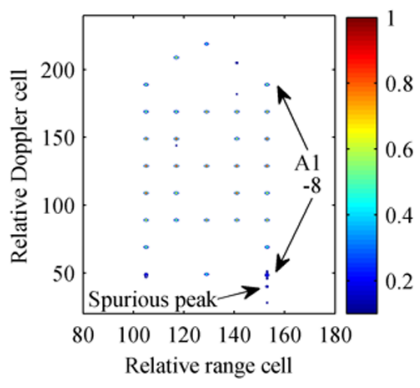

(a)

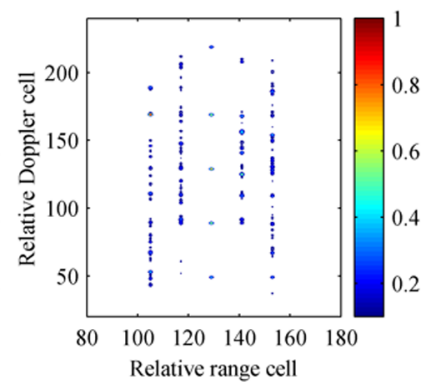

(b)

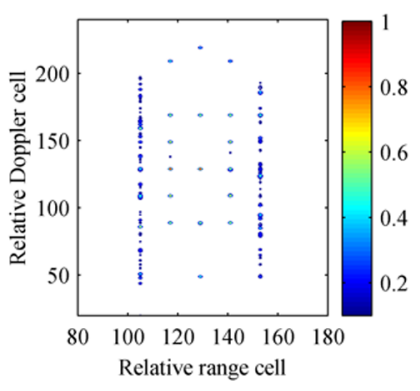

(c)

Fig. 5 Simulation results: (a) result of LPAF-based ISAR imaging algorithm, (b) result of LPWDbased ISAR imaging algorithm, and (c) result of MCPF-based ISAR imaging algorithm. 


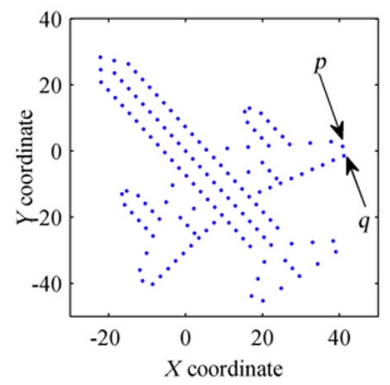

(a)

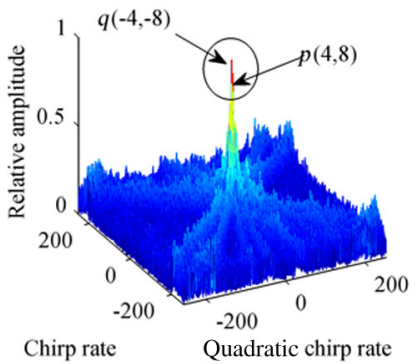

(d)

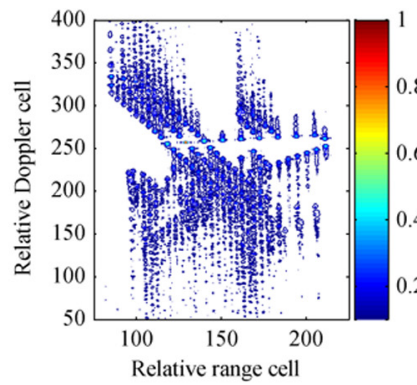

(b)

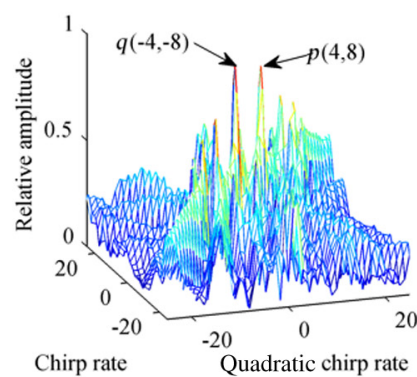

(e)

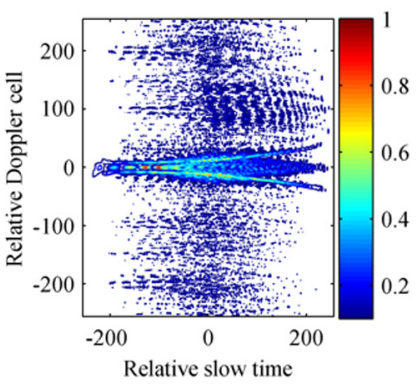

(c)

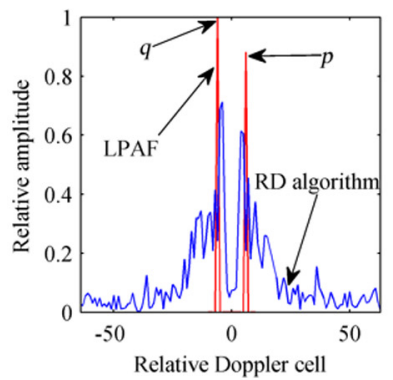

(f)

Fig. 6 (a) Aircraft target model, (b) result of the conventional RD algorithm, (c) WVD of the extracted data, (d) simulation result with the proposed algorithm, (e) zoomed-in plot of ellipse area in (d), and (f) result after the Doppler spread compensation with LPAF.

the 211st range bin. It is not difficult to find that the Doppler frequency is time-varying and nonlinear, which also demonstrates that the CPS model is very appropriate. Here we apply the LPAF-based parameter estimation algorithm to the extracted data. In Figs. 6(d) and 6(e), two peaks appear and can be easily picked out. Then the corresponding CR and QCR of two peaks can be correctly estimated via peak detection technique and CLEAN technique.

With the parameter estimation algorithm, we can estimate $\left(\phi_{p, 2}, \phi_{p, 3}\right)$ and $\left(\phi_{q, 2}, \phi_{q, 3}\right)$ as $\left(4 \mathrm{~Hz} / \mathrm{s}, 8 \mathrm{~Hz} / \mathrm{s}^{2}\right)$ and $\left(-4 \mathrm{~Hz} / \mathrm{s},-8 \mathrm{~Hz} / \mathrm{s}^{2}\right)$, respectively. After the Doppler spread compensation with the estimated parameters, we obtain the results in Fig. 6(f) by performing FFT, where the results of the conventional RD algorithm are also provided. Simulation shows that signal energy cannot be focused efficiently for Doppler frequency shift induced by $\mathrm{CR}$ and QCR. Consequently, the effective parameter estimation and compensation are quite essential.

Generally speaking, the better the focused quality of image, the smaller is the entropy. ${ }^{5,10,11,13,14}$ As before, the entropy is applied to measure the quality of the image.

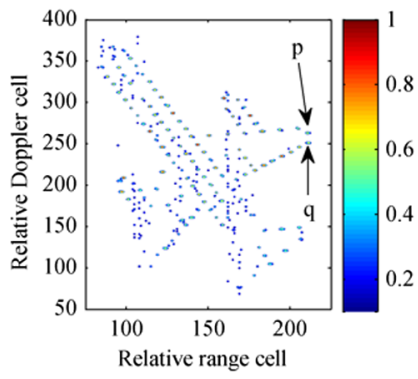

(a)

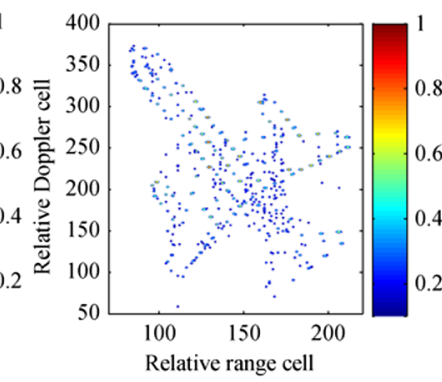

(b)

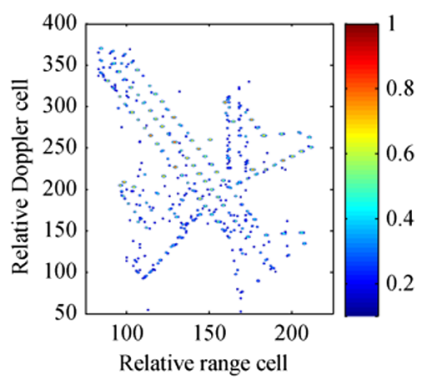

(c)

Fig. 7 Simulation results: (a) result of LPAF-based ISAR imaging algorithm, (b) result of LPWDbased ISAR imaging algorithm, and (c) result of MCPF-based ISAR imaging algorithm. 
Table 7 Entropies of ISAR images in Fig. 7.

\begin{tabular}{llll}
\hline \hline & Fig. 7(a) & Fig. 7(b) & Fig. 7(c) \\
\hline Entropies & 5.6388 & 5.9089 & 5.8016 \\
\hline \hline
\end{tabular}

Compared with the LPWD-based imaging algorithm and the MCPF-based imaging algorithm, this proposed LPAF-based imaging algorithm can obtain robust performance against noise. Therefore, most scatterers have been reconstructed correctly in Fig. 7(a) and spurious scatterers in Fig. 7(a) are fewer than those in Figs. 7(b) and 7(c). Also, the entropy of image in Fig. 7(a) is smaller than those in other figures in Table 7, which indicates that the image quality for the LPAF-based ISAR imaging algorithm is better than those for other algorithms.

In order to further validate the effectiveness of the LPAF-based ISAR imaging algorithm for targets with complex motion, we provide the results of the LPAF-based ISAR imaging algorithm under a range of different SNRs in Fig. 8, where the scope of $\mathrm{SNR}_{\text {in }}$ is from 2 to $-8 \mathrm{~dB}$ with an increment of $-2 \mathrm{~dB}$. It is worthwhile to mention that the less spurious scatterers appear in Figs. 8(a)-8(c), and the entropies of Figs. 8(a)-8(c) are small than those of Figs. 8(d)-8(f) in Table 8. Especially, the images in Figs. 8(e) and 8(f) are blurred severely by spurious scatterers. Thus, we conclude that the proposed ISAR imaging algorithm fails when the input SNR falls below $-4 \mathrm{~dB}$.

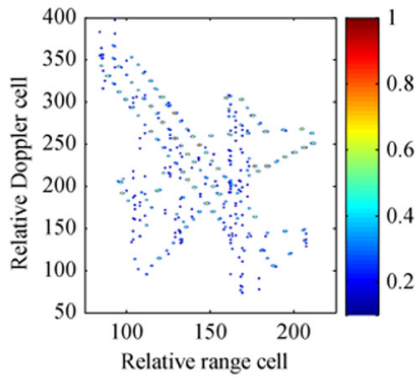

(a)

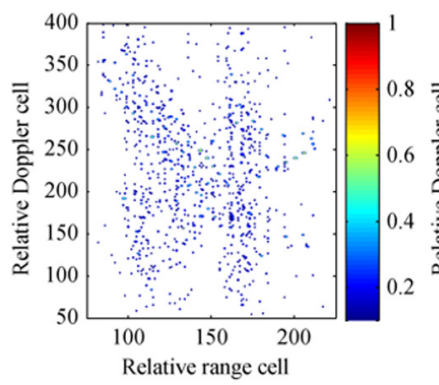

(d)

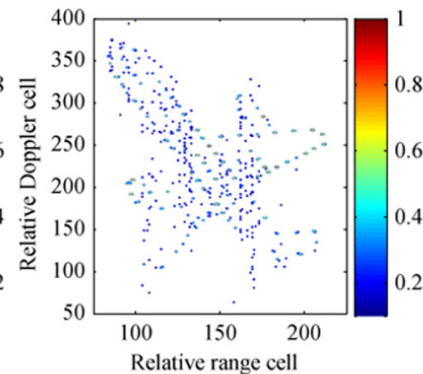

(b)

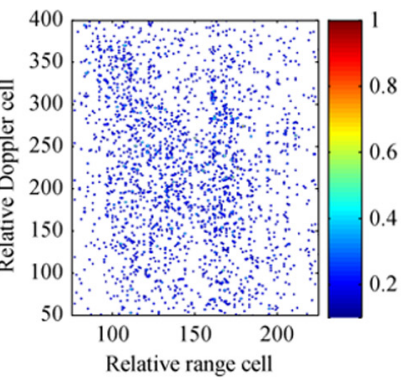

(e)

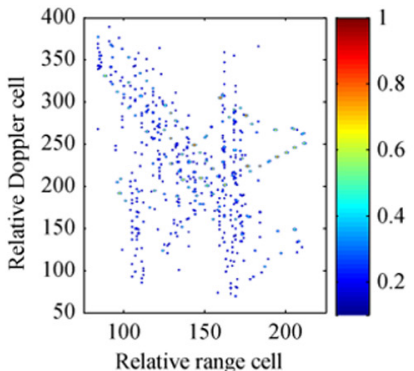

(c)

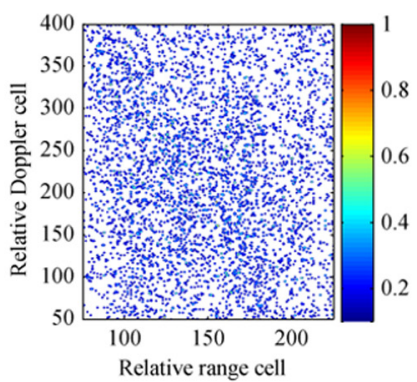

(f)

Fig. 8 Result of LPAF-based ISAR imaging algorithm under the situation of (a) $\mathrm{SNR}_{\text {in }}=2 \mathrm{~dB}$, (b) $\mathrm{SNR}_{\text {in }}=0 \mathrm{~dB}, \quad$ (c) $\mathrm{SNR}_{\text {in }}=-2 \mathrm{~dB}, \quad$ (d) $\mathrm{SNR}_{\text {in }}=-4 \mathrm{~dB}, \quad$ (e) $\mathrm{SNR}_{\text {in }}=-6 \mathrm{~dB}$, and (f) $\mathrm{SNR}_{\text {in }}=-8 \mathrm{~dB}$.

Table 8 Entropies of ISAR images in Fig. 8.

\begin{tabular}{lllllll}
\hline \hline & Fig. 8(a) & Fig. 8(b) & Fig. 8(c) & Fig. 8(d) & Fig. 8(e) & Fig. 8(f) \\
\hline Entropies & 5.6527 & 5.7258 & 5.8516 & 6.5244 & 7.9125 & 8.9486 \\
\hline \hline
\end{tabular}




\section{Conclusion}

For ISAR imaging of targets with complex motion, the azimuth echo in a range bin can be modeled as multicomponent CPSs, which have been proved more appropriate than the LFM model. This paper presents a new ISAR imaging algorithm for targets with complex motion based on LPAF. LPAF can directly obtain CRQCRD only by FFT operation, where two parameters can be estimated simultaneously via peak detection. With the analyses and the simulations, we demonstrate that the LPAF outperforms MCPF and LPWD for the parameter estimation of CPS in the robustness of performance against noise, the computational cost, and the cross-term suppression. Finally, the effectiveness of the proposed ISAR imaging algorithm is validated by the experimental results of simulated data.

\section{Acknowledgments}

This work was supported in part by the National Natural Science Foundation of China under Grant Nos. 61271024 and 61201292 and in part by the Science and Technology Foundation of Shaanxi Province under Grant 2012JM8015. Qian Lv conceived the work in this paper that led to the submission, experiment design, and accomplishment of the writing of the manuscript. Jibin Zheng played an important role in revising the manuscript and providing English language support. Tao $\mathrm{Su}$ is mainly responsible for approving the final version.

\section{References}

1. F. Berizzi et al., "High-resolution ISAR imaging of maneuvering targets by means of the range instantaneous Doppler technique: modeling and performance analysis," IEEE Trans. Image Process. 10(12), 1880-1890 (2001).

2. Y. Li et al., "Inverse synthetic aperture radar imaging of ship target with complex motion," IET Radar Sonar Navig. 2(6), 395-403 (2008).

3. J. Wood and D. Barry, "Radon transformation of time-frequency distributions for analysis of multicomponent signals," IEEE Trans. Signal Process. 42(11), 3166-3177 (1994).

4. Y. Wang, "Inverse synthetic aperture radar imaging of manoeuvring target based on rangeinstantaneous-Doppler and range-instantaneous-chirp-rate algorithms," IET Radar Sonar Navig. 6(9), 921-928 (2012).

5. J. Zheng et al., "ISAR imaging of targets with complex motions based on the keystone timechirp rate distribution," IEEE Geosci. Remote Sens. Lett. 11(7), 1275-1279 (2014).

6. M. Xing et al., "New ISAR imaging algorithm based on modified Wigner-Ville distribution," IET Radar Sonar Navig. 3(1), 70-80 (2009).

7. X. Lv et al., "ISAR imaging of maneuvering targets based on the range centroid Doppler technique," IEEE Trans. Image Process. 19(1), 141-153 (2010).

8. X. Lv et al., "Lv's distribution: principle, implementation, properties, and performance," IEEE Trans. Signal Process. 59(8), 3576-3591 (2011).

9. R. Tao, N. Zhang, and Y. Wang, "Analysing and compensating the effects of range and Doppler frequency migrations in linear frequency modulation pulse compression radar," IET Radar, Sonar Navig. 5(1), 12-22 (2011).

10. X. Bai et al., "ISAR imaging of a ship target based on parameter estimation of multicomponent quadratic frequency-modulated signals," IEEE Trans. Geosci. Remote Sens. 52(2), 1418-1429 (2014).

11. J. Zheng et al., "ISAR imaging of targets with complex motion based on the chirp ratequadratic chirp rate distribution," IEEE Trans. Geosci. Remote Sens. 52(11), 7276-7289 (2014).

12. Y. Li et al., "A new algorithm of ISAR imaging for maneuvering targets with low SNR," IEEE Trans. Aerosp. Electron. Syst. 49(1), 543-557 (2013).

13. J. Zheng et al., "ISAR imaging of nonuniformly rotating target based on a fast parameter estimation algorithm of cubic phase signal," IEEE Trans. Geosci. Remote Sens. 53(9), 4727-4740 (2015). 
Lv, Su, and Zheng: Inverse synthetic aperture radar imaging of targets with complex motion based...

14. Y. Wang and B. Zhao, "Inverse synthetic aperture radar imaging of nonuniformly rotating target based on the parameters estimation of multicomponent quadratic frequency-modulated signals," IEEE Sensors J. 15(7), 4053-4061 (2015).

15. P. O'shea, "A fast algorithm for estimating the parameters of a quadratic FM signal," IEEE Trans. Signal Process. 52(2), 385-393 (2004).

16. P. O'shea, “A new technique for instantaneous frequency rate estimation," IEEE Signal Process. Lett. 9(8), 251-252 (2002).

17. S. Peleg and B. Porat, "Linear FM signal parameter estimation from discrete-time observations," IEEE Trans. Aerosp. Electron. Syst. 27(4), 607-616 (1991).

18. Y. Wang and Y. Jiang, "Inverse synthetic aperture radar imaging of maneuvering target based on the product generalized cubic phase function," IEEE Geosci. Remote Sens. Lett. 8(5), 958-962 (2011).

19. Y. Wang and Y. Jiang, "ISAR imaging of a ship target using product high-order matchedphase transform," IEEE Geosci. Remote Sens. Lett. 6(4), 658-661 (2009).

20. L. Stankovic, "Local polynomial Wigner distribution," Signal Process. 59(1), 123-128 (1997).

21. Y. Wang, J. Kang, and Y. Jiang, "ISAR imaging of maneuvering target based on the local polynomial Wigner distribution and integrated high-order ambiguity function for cubic phase signal model," IEEE J. Sel. Top. Appl. Earth Obs. Remote Sens. 7(7), 2971-2991 (2014).

22. Y. Wang, B. Zhao, and J. Kang, "Asymptotic statistical performance of local polynomial Wigner distribution for the parameters estimation of cubic-phase signal with application in ISAR imaging of ship target," IEEE J. Sel. Top. Appl. Earth Obs. Remote Sens. 8(3), 10871098 (2015).

23. L. Wu et al., "ISAR imaging of targets with complex motion based on discrete chirp Fourier transform for cubic chirps," IEEE Trans. Geosci. Remote Sens. 50(10), 42014212 (2012).

24. M. Xing, R. Wu, and Z. Bao, "High resolution ISAR imaging of high speed moving targets," IEE Proc. Radar Sonar Navig. 152(2), 58-67 (2005).

25. D. Wahl et al., "Phase gradient autofocus - a robust tool for high resolution SAR phase correction," IEEE Trans. Aerosp. Electron. Syst. 30(3), 827-835 (1994).

26. Y. Li et al., "Inverse synthetic aperture radar imaging of targets with nonsevere maneuverability based on the centroid frequency chirp rate distribution," J. Appl. Remote Sens. 9, 095065 (2015).

27. S. Barbarossa, A. Scaglione, and G. B. Giannakis, "Product high-order ambiguity function for multicomponent polynomial-phase signal modeling," IEEE Trans. Signal Process. 46(3), 691-708 (1998).

28. X. Xia, "Discrete chirp-Fourier transform and its application to chirp rate estimation," IEEE Trans. Signal Process. 48(11) 3122-3133 (2000).

29. X. Guo et al., "Comments on discrete chirp-Fourier transform and its application to chirp rate estimation," IEEE Trans. Signal Process. 50(12), 3115-3116 (2002).

30. S. Peleg and B. Porat, "The Cramer-Rao lower bound for signals with constant amplitude and polynomial phase," IEEE Trans. Signal Process. 39(3), 749-752 (1991).

31. P. Wang et al., "Integrated cubic phase function for linear FM signal analysis," IEEE Trans. Aerosp. Electron. Syst. 46(3), 963-977 (2010).

Qian Lv received her BS degree in measuring and control techniques and instruments from Xi' an Shiyou University, Shaanxi, China, in 2013. She is currently working toward her PhD with the National Laboratory of Radar Signal Processing, Xidian University, Xi'an, China. Her research interests include synthetic aperture radar (SAR) and inverse SAR signal processing, time-frequency analysis, and interferometric inverse synthetic aperture radar (InISAR) image.

Tao Su received his BS degree in information theory, his MS degree in mobile communication, and his $\mathrm{PhD}$ in signal and information processing from Xidian University, Xi'an, China, in 1990, 1993, and 1999, respectively. Since 1993, he has been a professor with the National Laboratory 
of Radar Signal Processing, School of Electronic Engineering. His research interests include high-speed real-time signal processing on radar, sonar and telecommunications, digital signal processing, parallel processing system design, and FPGA IP design.

Jibin Zheng received his BS degree in electronic information science and technology from Shandong Normal University, Shandong, China, in 2009 and his $\mathrm{PhD}$ in signal and information processing from Xidian University, Xi'an, China, in 2015. From September 2012 to September 2014, he worked as a visiting PhD student at the Department of Electrical Engineering, Duke University, Durham, North Carolina. His research interests include SAR and inverse SAR signal processing, cognitive radar. 